\title{
Vortices with massive cores in a binary mixture of Bose-Einstein condensates
}

\author{
Andrea Richaud (1)* and Vittorio Penna \\ Dipartimento di Scienza Applicata e Tecnologia and u.d.r. CNISM, Politecnico di Torino, Corso Duca degli Abruzzi 24, I-10129 Torino, Italy \\ Ricardo Mayol and Montserrat Guilleumas \\ Departament de Física Quàntica i Astrofísica and Institut de Ciències del Cosmos, Facultat de Física, \\ Universitat de Barcelona, E-08028 Barcelona, Spain
}

(Received 17 September 2019; published 23 January 2020)

\begin{abstract}
We analyze a notable class of states relevant to an immiscible bosonic binary mixture loaded in a rotating boxlike circular trap, i.e., states where vortices in one species host the atoms of the other species, which thus play the role of massive cores. Within a fully analytical framework, we calculate the equilibrium distance distinguishing the motion of precession of two corotating massive vortices, the angular momentum of each component, the vortices healing length, and the characteristic size of the cores. We then compare these previsions with the measures extracted from the numerical solutions of the associated coupled Gross-Pitaevskii equations. Interestingly, making use of a suitable change of reference frame, we show that vortices drag the massive cores which they host thus conveying their same motion of precession, but that there is no evidence of tangential entrainment between the two fluids, since the cores keep their orientation constant while orbiting.
\end{abstract}

DOI: 10.1103/PhysRevA.101.013630

\section{INTRODUCTION}

Vortices in quantum fluids are topological excitations characterized by quantized circulation [1] which are present in a number of nonlinear field theories and models [2], ranging from superfluid media $[3,4]$ and quantum optics $[5,6]$ to superconductivity theories [7,8] and Josephson-junction arrays $[9,10]$, and play a key role in fundamental effects such as superfluid turbulence [11], the Berezinskii-KosterlitzThouless transition [12], fractional statistics [13], and in the development of a fully quantized field theory for topologically complex excitations [14-16]. Among the plethora of different physical systems where vortices can be experimentally investigated, ultracold quantum gases provide a particularly controllable and versatile platform $[17,18]$ for the study and the observation of the rich phenomenology associated to their formation [19,20], dynamics [21,22], and interactions [23]. Vortices in Bose-Einstein condensates (BECs) were first obtained by means of a phase-imprinting method involving two hyperfine spin states of ${ }^{87} \mathrm{Rb}$ [24] but, at present, can be produced also by stirring the BEC above a certain critical velocity [25-27], dragging barriers through the BEC itself [28] or interfering multiple condensate fragments [29].

Solitons are a kind of localized excitations which, because of the competition between dispersion and nonlinearity, propagate keeping their shape unaltered, even if a two-soliton collision occurs [30]. Soon after the achievement of BoseEinstein condensation, different types of solitons have been described and observed [18,31,32]. To our purposes, of particular importance are those systems where a bosonic binary

\footnotetext{
*Corresponding author: andrea.richaud@polito.it
}

mixture features dark-bright soliton configurations [33-35]. These structures, first predicted in Ref. [36], are frequently termed as symbiotic solitons [37] because the bright component, being endowed with repulsive intraspecies interaction, could not exist if the dark component did not play the role of an effective confining potential.

The same symbiotic relationship was shown to constitute the mechanism underlying the robustness of vortex-bright soliton complexes. First observed in 2000 by the JILA group [38], they represent the topological extension of the darkbright soliton configuration to the case where a component hosts one or more vortices [39]. The aforementioned study paved the way to a series of further investigations which highlighted, among various aspects, the spontaneous generation of vortex-bright soliton structures [40], the possibility, for the effective potential well corresponding to the vortex core, to support not only bound states [41], but also multiring excited radial state complexes [42], the splitting of doubly quantized composite vortices [43], and a rich dynamical scenario for the bright-solitary component [44].

Within an analytical framework and by means of extensive numerical simulations, our work aims at analyzing the static and the dynamical properties of vortex-bright soliton complexes, i.e., how the presence of massive solitons within the cores of two corotating vortices affect the equilibrium distance characterizing their motion of precession around the trap center, the role of the interspecies repulsion as an antagonist to the centrifugal force acting on the solitons, the functional dependence of the angular momenta carried by two species, of the vortex healing length, and of the characteristic radius of the massive cores on the mass of the latter.

If one considers repulsive intraspecies $\left(g_{a}, g_{b}\right)$ and interspecies $\left(g_{a b}\right)$ interactions such that the immiscibility 
condition $g_{a b}>\sqrt{g_{a} g_{b}}$ is fulfilled [45], the dynamical picture of the mixture [in which the order-parameter fields of the two species obey two coupled Gross-Pitaevskii equations (GPEs)], indeed reduces to the much simpler equations of two pointlike vortices with nonzero-mass cores. Noticeably, the latter are found to exhibit an evident Lorentz-like form since, in the presence of vortex cores occupied by a second species, the vortex-motion equations are equivalent to those of a pair of massive charges acted by a transverse magnetic field. With negligible fractions of the minority component, one recovers the Helmholtz-Kirchhoff equations for planar pointlike vortices [46].

The outline of the paper is the following: in Sec. II, we present an analytical model for the dynamics of massive vortices in a confined system which incorporates the effect of the virtual vortices resulting from the boundary condition of vanishing normal velocity. In particular, we derive a formula giving the equilibrium distance distinguishing the motion of precession of two corotating massive vortices. Section III is devoted to the presentation of the two coupled stationary GPEs which provide a good description of the bosonic binary mixture in the mean-field approximation. In Sec. IV, we show how the presence of massive cores (i.e., species- $b$ atoms trapped within species- $a$ vortices) affects the equilibrium distance of the pair of corotating vortices. We also show that the interspecies repulsion tends to counterbalance the centrifugal force acting on species- $b$ atoms. In Sec. V, we address the angular momenta of the two components and provide analytical formulas that well capture their functional dependence on the number of species- $b$ atoms (which, in turn, is directly proportional to the mass of the cores). By means of a suitable change of reference frame, we show that the cores, although following the same motion of precession of the vortices, keep their orientation constant while orbiting. This circumstance witnesses the fact that there is no tangential entrainment between the two fluids. In Sec. VI, we present an heuristic but effective system of equations that well reproduces the functional dependence of the vortex healing lengths and of the cores' characteristic radius on the number of species- $b$ atoms. Eventually, Sec. VII is devoted to concluding remarks.

\section{POINTLIKE VORTICES IN A CIRCULAR BOX}

In this section, we review some results concerning the dynamics of pointlike vortices and we introduce a model for the dynamics of vortices whose cores host pointlike masses (hence the name massive vortices, as opposed to the traditional massless vortices).

\section{A. Massless vortices}

The Hamiltonian of $N$ pointlike massless vortices in an ideal unbounded fluid is given by [46]

$$
H_{\infty}=\left(z_{1}, \ldots, z_{N}\right)=-\frac{\rho_{*}}{4 \pi} \sum_{i=1}^{N} \sum_{j \neq i} k_{i} k_{j} \ln \frac{\left|z_{i}-z_{j}\right|}{\lambda},
$$

where $\rho_{*}$ is the fluid planar density, $z_{j}=x_{j}+i y_{j} \in \mathbb{C}$ is the position of the $j$ th vortex in the ambient plane, and $k_{j}=$ $n_{j} h / m_{f}$ is its strength $\left(n_{j} \in \mathbb{Z}\right.$ is the vortex quantization and $m_{f}$ is the mass of the fluid particles). In the following, we will specialize our discussion to the case of $N=2$ vortices.

When one considers bounded systems, Hamiltonian (1) modifies due to the presence of the confining potential. In the case of a boxlike potential (this type of confinement is within the reach of current experimental trapping techniques, see, e.g., Refs. [47-49]), the presence of a boundary confining the fluid is accounted for by means of the virtual charge method, i.e., by introducing a suitable configuration of virtual vortices. With this premise in mind, the Hamiltonian of $N=2$ pointlike massless vortices in an ideal fluid confined in a circular box of radius $R$ reads [50]

$$
\begin{aligned}
H= & \frac{\rho_{*}}{4 \pi}\left\{k_{1} k_{2} \ln \frac{\left|R^{2}-z_{1} \bar{z}_{2}\right|^{2}}{\left|R\left(z_{1}-z_{2}\right)\right|^{2}}\right. \\
& \left.+k_{1}^{2} \ln \left(1-\frac{\left|z_{1}\right|^{2}}{R^{2}}\right)+k_{2}^{2} \ln \left(1-\frac{\left|z_{2}\right|^{2}}{R^{2}}\right)\right\}
\end{aligned}
$$

In this framework, the coordinates of each vortex constitute a pair of canonically conjugate variables, and motion equations can be obtained by means of the Poisson brackets,

$$
\{F, G\}=\frac{1}{\rho_{*} k_{j}} \sum_{j=1}^{2}\left[\frac{\partial F}{\partial x_{j}} \frac{\partial G}{\partial y_{j}}-\frac{\partial G}{\partial x_{j}} \frac{\partial F}{\partial y_{j}}\right],
$$

involving, in turn, the canonical brackets $\left\{x_{i}, y_{j}\right\}=\delta_{i, j} /\left(\rho_{*} k_{j}\right)$ (see, for example, Ref. [51]).

\section{B. Massive vortices}

If one wants to introduce into the model the fact that the vortex cores host point masses, it is convenient to move to the Lagrangian formalism, where the presence of massive cores can be taken into account as follows:

$$
L=\sum_{j=1}^{2}\left[\frac{m_{j}}{2}\left(\dot{x}_{j}^{2}+\dot{y}_{j}^{2}\right)+\frac{k_{j} \rho_{*}}{2}\left(y_{j} \dot{x}_{j}-x_{j} \dot{y}_{j}\right)\right]-H,
$$

where $m_{j}$ represents the pointlike mass hosted by the $j$ th vortex core and where $\dot{q}_{j}:=d q_{j} / d t$ (with $q=x, y$ ). Note that, Lagrangian (3) is formally equivalent to that describing charged particles in a planar domain subject to a transverse magnetic field, where $k_{j} \mathrm{~s}$ and $\rho_{*}$ play the roles of charges and of magnetic field, respectively. As is well known, the dynamics of the vortex cores is generated by the EulerLagrange equations which, in the special but interesting case of two equal vortices $k_{1}=k_{2}=k$, whose cores host two equal masses $m_{1}=m_{2}=m$, take the form

$$
\begin{aligned}
m \ddot{\vec{r}}_{j}= & k \rho_{*} \vec{u}_{3} \wedge \dot{\vec{r}}_{j}+\rho_{*} \frac{k^{2}}{2 \pi}\left[\frac{\vec{r}_{j}-\vec{r}_{i}}{\left|\vec{r}_{j}-\vec{r}_{i}\right|^{2}}+\frac{\vec{r}_{j}}{R^{2}-r_{j}^{2}}\right. \\
& \left.+\frac{R^{2} \vec{r}_{i}-r_{i}^{2} \vec{r}_{j}}{R^{4}-2 R^{2} \vec{r}_{i} \vec{r}_{j}+r_{i}^{2} r_{j}^{2}}\right]
\end{aligned}
$$

for $i, j \in\{1,2\}$ and $i \neq j\left[\vec{u}_{3}\right.$ is the unit vector perpendicular to the plane $(x, y)]$. 
The resulting system of four differential equations admits a notable solution,

$$
\begin{aligned}
& x_{1}(t)=\frac{d}{2} \cos (\Omega t), \quad y_{1}(t)=\frac{d}{2} \sin (\Omega t) \\
& x_{2}(t)=\frac{d}{2} \cos (\Omega t+\pi), \quad y_{2}(t)=\frac{d}{2} \sin (\Omega t+\pi),
\end{aligned}
$$

provided that the two vortices are placed symmetrically with respect to the box-trap center and that their distance $d$ and the angular frequency $\Omega$ marking their motion of precession fulfill the following equation:

$$
\begin{aligned}
\frac{\pi d^{6} \Omega\left(k \rho_{*}-m \Omega\right)+3 d^{4} k^{2} \rho_{*}}{d-2 R} \\
=-\frac{16 \pi d^{2} R^{4} \Omega\left(m \Omega-k \rho_{*}\right)+16 k^{2} \rho_{*} R^{4}}{d-2 R} .
\end{aligned}
$$

As expected, Eq. (4) shows a pathology when $d \rightarrow 2 R$, meaning that the vortex pair is approaching the circular-box boundary. Moreover, in the limit of infinite box radius $(R \rightarrow$ $+\infty$ ), one can retain only those terms $\propto R^{4}$ and the relation $d(\Omega)$ can be expressed in closed form, i.e.,

$$
d=k \sqrt{\frac{\rho_{*}}{\pi}} \frac{1}{\sqrt{k \rho_{*} \Omega-m \Omega^{2}}} .
$$

In Sec. IV A, the equilibrium distance $d$ predicted by Eq. (4) and relevant to two equal pointlike massive vortices in a circular box will be compared to the one obtained by numerically solving two coupled stationary GPEs. To conclude this section, we would like to remark that the extension of model (3) to the case of harmonic confinement is far from being trivial, as the unavoidable curvature of the enveloping wave function produces non-negligible effective forces acting on the vortices' centers, which distort the usual vortex dynamics already in the case of zero species- $b$ atoms. It is indeed the use of a boxlike potential that allows one to bypass the influence of the aforementioned non-negligible effective forces, thus allowing for a cleaner emergence of the genuine phenomenology characterizing vortex/bright-soliton complexes.

\section{THE BOSONIC MIXTURE}

We consider a bosonic mixture of ${ }^{23} \mathrm{Na}$ and ${ }^{39} \mathrm{~K}[52,53]$. Each atomic species is characterized by an order parameter, $\varphi_{a}=\sqrt{N_{a}} \psi_{a}$ and $\varphi_{b}=\sqrt{N_{b}} \psi_{b}$, respectively. In a mean-field treatment of the problem, we assume that the system is effectively quasi-2D, as a result of a strong confinement along the $z$ direction. Because of this, it can be effectively modeled by the following two coupled stationary GPEs:

$$
\begin{gathered}
-\frac{\hbar^{2}}{2 m_{a}}\left[\frac{\partial^{2}}{\partial x^{2}}+\frac{\partial^{2}}{\partial y^{2}}\right] \psi_{a}+\frac{g_{a} N_{a}}{\ell_{z}}\left|\psi_{a}\right|^{2} \psi_{a} \\
+\frac{g_{a b} N_{b}}{\ell_{z}}\left|\psi_{b}\right|^{2} \psi_{a}+V_{\mathrm{ext}, a} \psi_{a}=\mu_{a} \psi_{a} \\
-\frac{\hbar^{2}}{2 m_{b}}\left[\frac{\partial^{2}}{\partial x^{2}}+\frac{\partial^{2}}{\partial y^{2}}\right] \psi_{b}+\frac{g_{b} N_{b}}{\ell_{z}}\left|\psi_{b}\right|^{2} \psi_{b} \\
+\frac{g_{a b} N_{a}}{\ell_{z}}\left|\psi_{a}\right|^{2} \psi_{b}+V_{\mathrm{ext}, b} \psi_{b}=\mu_{b} \psi_{b},
\end{gathered}
$$

where $N_{a}\left(N_{b}\right)$ corresponds to the number of species- $a$ (species-b) atoms, $g_{c}=4 \pi \hbar^{2} a_{c} / m_{c}$, with $c=a, b$ are the intraspecies interaction strengths and $g_{a b}=2 \pi \hbar^{2} a_{a b} / m_{a b}$ is the interspecies coupling. Notice that $m_{a}\left(m_{b}\right)$ is the atomic mass of sodium (potassium), while $m_{a b}=\left(m_{a}^{-1}+m_{b}^{-1}\right)^{-1}$ is their reduced mass; similarly, $a_{a}$ and $a_{b}$ are the intraspecies scattering lengths, while $a_{a b}$ is the interspecies scattering length. Parameter $\ell_{z}$ is the effective thickness of the disklike box trap and functions $\psi_{a}$ and $\psi_{b}$ are normalized to 1 , since $\varphi_{a}$ and $\varphi_{b}$ are, respectively, normalized to $N_{a}$ and $N_{b}$.

Vortical solutions of Eqs. (6) are found by moving to a frame rotating with angular velocity $\Omega$ (this corresponds to adding the term $-\Omega \hat{L}_{z}$ to the Hamiltonian, where $\hat{L}_{z}$ is the operator associated to the third component of the angular momentum) and then employing the imaginary-time method $[41,54]$. The starting condition for the imaginary-time dynamics is such that species- $a$ hosts a vortex pair while species- $b$ is localized (two narrow Gaussian distributions) at the vortex cores. As the fictitious dynamics advances, the position of the vortex cores, their healing length, together with the spatial distribution of species- $b$ atoms is iteratively self-consistently refined, until convergence is reached. We conclude this section by noticing that, for our purposes, solutions of Eqs. (6) of the type vortex/bright-soliton pairs (see, e.g., Fig. 1) could be either actual ground states or excited metastable states (i.e., either global or local minima in the energy landscape).

\section{MASSIVE VORTEX PAIRS IN A BINARY MIXTURE OF BECS}

Eigensystem (6) was solved sweeping model parameter $N_{b}$, the number of species- $b$ atoms, which constitute the massive cores of species- $a$ vortices. As explained in Sec. III, a suitable ansatz for the starting condition of the imaginary-time dynamics was chosen. In the whole range of $N_{b}$ that we explored (i.e., $\left.N_{b} \in[5,1000]\right)$, our numerical simulations [55] converged to a stationary state of the type illustrated in Fig. 1.

Basically, condensate $a$ is highly confined by the boxlike potential (whose radius is $R=50 \mu \mathrm{m}$ ) and is marked by the presence of two corotating vortices. The latter are symmetrically positioned with respect to the center of the trap; they are such that the density $\left|\psi_{a}\right|^{2}$ goes to zero in the center of the cores and features a quantized circulation. On the other hand, component $b$ occupies the vortex cores which, in turn, constitute an effective double-well potential for species- $b$ atoms [44].

One can gain further insight into the discussed eigensolution by computing the mass current density

$$
\vec{J}_{c}=-\frac{i \hbar}{2}\left(\psi_{c}^{*} \nabla \psi_{c}-\psi_{c} \nabla \psi_{c}^{*}\right),
$$

(with $c=a, b$ ) which also corresponds to the momentumper-particle distribution. As illustrated in the second row of Fig. 1, both vortices in species $a$ rotate anticlockwise, thus determining a collective motion of precession which is anticlockwise too. As concerns species- $b$ atoms, they are dragged by condensate $a$ and remain bound within the vortex cores, thus featuring their same motion of precession around the center of the trap. With reference to the middle right panel of Fig. 1, one can appreciate that the left (right) peak of 

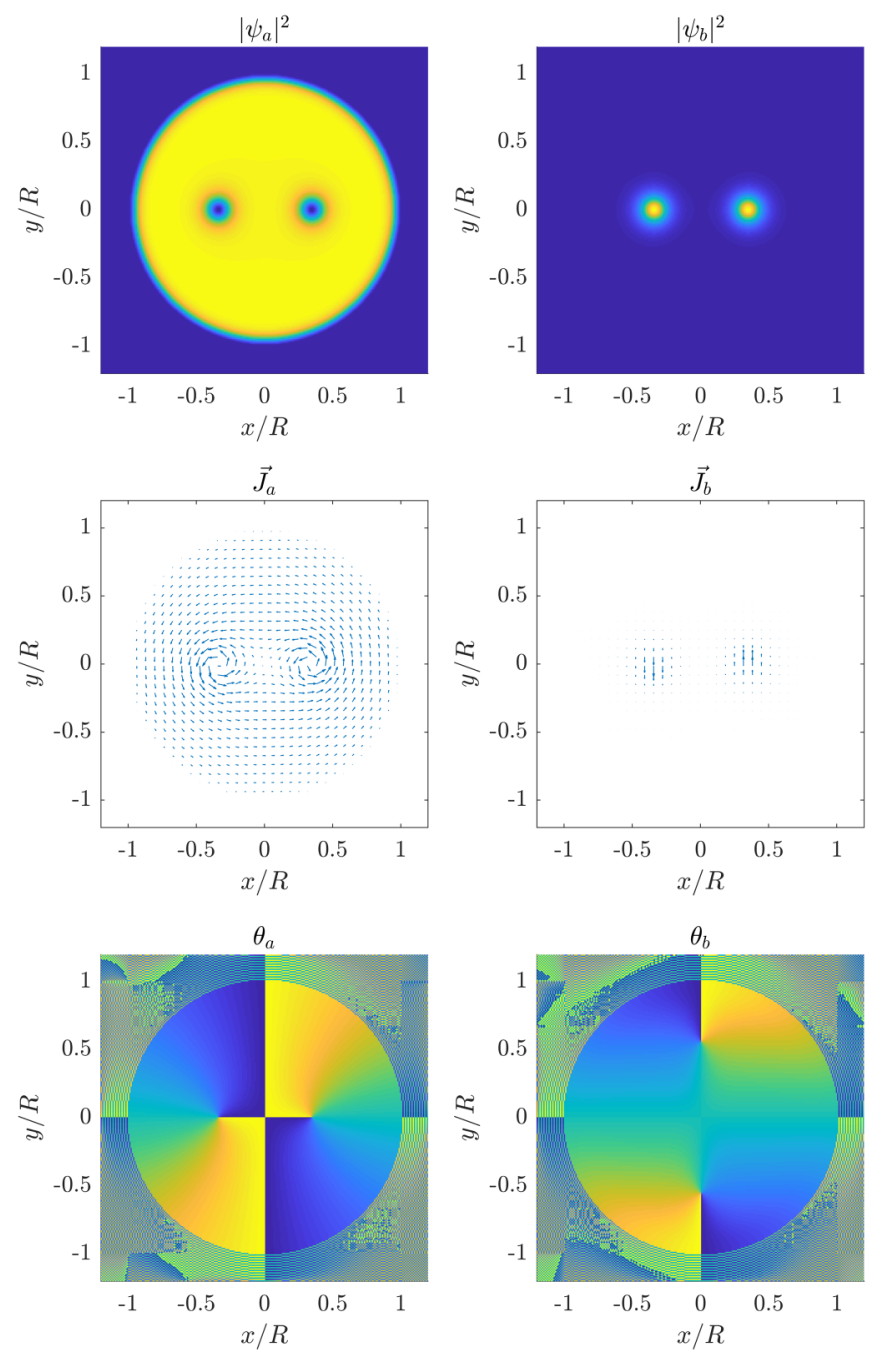

FIG. 1. Typical minimum-energy solution of eigensystem (6). First (second) row corresponds to the square moduli [mass current density (see Eq. 7)] of the eigensolutions $\psi_{a}$ and $\psi_{b}$. In the first row, yellow (blue) is associated to large (zero) values of the density $|\psi|^{2}$. Third row corresponds to the phase-field associated to $\psi_{a}$ and $\psi_{b}$ [blue (yellow) color corresponds to $-\pi(+\pi)$ ]. Left (right) column corresponds to species $a(b)$. The following parameters have been used: $N_{a}=5 \times 10^{4}, N_{b}=10^{3}, \Omega=5 \mathrm{rad} / \mathrm{s}, R=50 \mu \mathrm{m}, m_{a}=$ $3.82 \times 10^{-26} \mathrm{~kg}, m_{b}=6.48 \times 10^{-26} \mathrm{~kg}, g_{a}=52 \times\left(4 \pi \hbar^{2} a_{0}\right) / m_{a}$, $g_{b}=7.6 \times\left(4 \pi \hbar^{2} a_{0}\right) / m_{b}, g_{a b}=24.2 \times\left(2 \pi \hbar^{2} a_{0}\right) / m_{a b}, \ell_{z}=2 \mu \mathrm{m}$.

$\left|\psi_{b}\right|^{2}$ is translating downward (upward), i.e., along a direction tangential to the precession orbit.

Eventually, other important information that can be extracted from the eigensolutions $\psi_{a}$ and $\psi_{b}$ of equations (6) concerns the phase fields. The latter, denoted by $\theta_{a}$ and $\theta_{b}$, and such that $\psi_{c}=\sqrt{\left|\psi_{c}\right|^{2}} e^{i \theta_{c}}$ (where $c=a, b$ ), have been plotted in the lower panels of Fig. 1. As expected, $\theta_{a}$ features singularities in correspondence of the vortices' centers, while, less expectedly, $\theta_{b}$ features singularities too. Nevertheless, the latter are found far from the peaks of $\left|\psi_{b}\right|^{2}$. According to the basic properties of quantum fluids, the circulation

$$
\mathcal{C}_{\gamma}\left[\vec{v}_{c}\right]=\oint_{\gamma} \vec{v}_{c} \cdot d \vec{r}, \quad(c=a, b)
$$
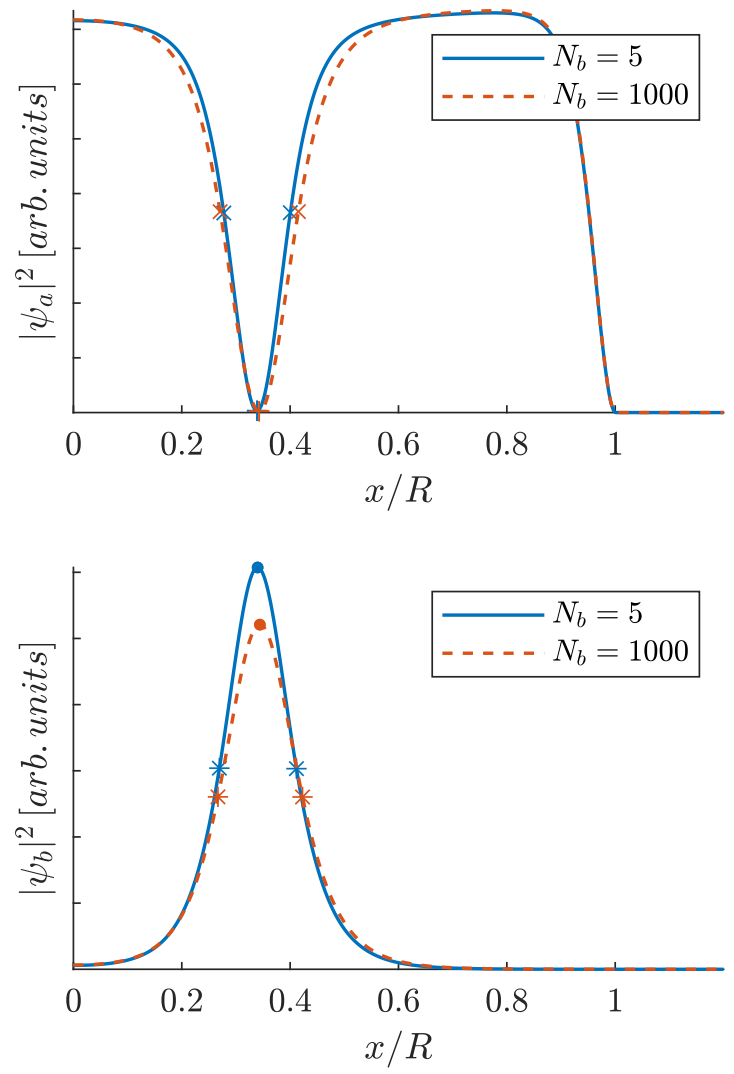

FIG. 2. Density profile of the minimum-energy solutions of eigensystem (6) along the axis $y=0$ (we have plotted just the range $x>0$ because both $\left|\psi_{a}\right|^{2}$ and $\left|\psi_{b}\right|^{2}$ are symmetric with respect to $x=0$ ) for two different values of $N_{b}$. Upper panel: The position of markers + corresponds to $d_{\mathrm{vor}} / 2$, while the distance between markers $\times$ corresponds to $2 \xi_{a}$. Lower panel: The position of markers $\times$ corresponds to $d_{\text {peak }} / 2$, while the distance between markers $*$ corresponds to $2 \sigma_{b}$. The following parameters have been used: $N_{a}=5 \times$ $10^{4}, \Omega=5 \mathrm{rad} / \mathrm{s}, R=50 \mu \mathrm{m}, m_{a}=3.82 \times 10^{-26} \mathrm{~kg}, m_{b}=6.48 \times$ $10^{-26} \mathrm{~kg}, g_{a}=52 \times\left(4 \pi \hbar^{2} a_{0}\right) / m_{a}, g_{b}=7.6 \times\left(4 \pi \hbar^{2} a_{0}\right) / m_{b}, g_{a b}=$ $24.2 \times\left(2 \pi \hbar^{2} a_{0}\right) / m_{a b}, \ell_{z}=2 \mu \mathrm{m}$.

of the velocity vector field associated to $\psi_{c}$, which means $\vec{v}_{c}=\frac{\hbar}{m_{c}} \nabla \theta_{c}$ (with $c=a, b$ ), is zero if the closed path $\gamma$ does not encircle singularities of the phase field $\theta_{c}$ (with $c=a, b$ ). Conversely, due to the Feynman-Onsager quantization rules, it takes values $n h / m_{c}$, where $n \in \mathbb{Z}$, if one or more singularities of the associated field $\theta_{c}$ are encircled by $\gamma$. This information will be useful to better understand the discussion about the angular momentum of species- $b$ bosons (see Sec. V B).

\section{A. Mass of the cores and equilibrium distance}

Increasing the number of species- $b$ atoms (within the investigated range $\left.N_{b} \in[5,1000]\right)$, the distance $d_{\text {vor }}$ between the centers of the vortices increases. Similarly, the distance between the two peaks of $\left|\psi_{b}\right|^{2}, d_{\text {peak }}$, increases upon increasing $N_{b}$. Figure 2 shows how the presence of massive cores deforms and displaces the vortices. Notice that the position of the peaks of $\left|\psi_{b}\right|^{2}$ does not exactly match that of the minima of $\left|\psi_{a}\right|^{2}$ due to the centrifugal force on species- $b$ atoms and the finite repulsive coupling $\left(g_{a b}<+\infty\right)$ between the two fluids which, 


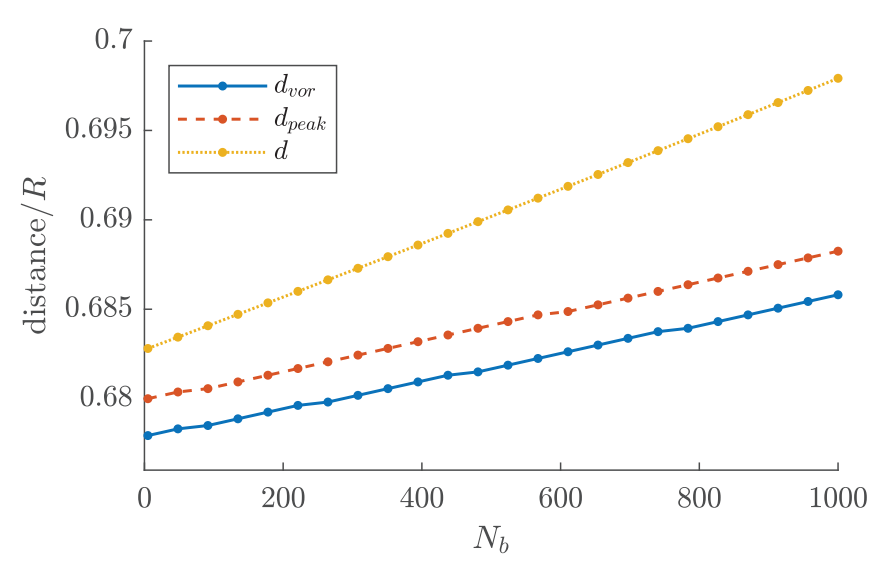

FIG. 3. Equilibrium distance: comparison between numerical $\left(d_{\mathrm{vor}}\right.$ and $\left.d_{\text {peak }}\right)$ and analytical $(d)$ results. The following parameters have been used: $N_{a}=5 \times 10^{4}, N_{b} \in[5,1000], \Omega=$ $5 \mathrm{rad} / \mathrm{s}, R=50 \mu \mathrm{m}, m_{a}=3.82 \times 10^{-26} \mathrm{~kg}, m_{b}=6.48 \times 10^{-26} \mathrm{~kg}$, $g_{a}=52 \times\left(4 \pi \hbar^{2} a_{0}\right) / m_{a}, \quad g_{b}=7.6 \times\left(4 \pi \hbar^{2} a_{0}\right) / m_{b}, \quad g_{a b}=24.2 \times$ $\left(2 \pi \hbar^{2} a_{0}\right) / m_{a b}, \ell_{z}=2 \mu \mathrm{m}$.

in turn, allows for a nonzero penetration of fluid $b$ into fluid $a$. Therefore, observables $d_{\text {vor }}$ and $d_{\text {peak }}$, which in the analytical model based on pointlike vortices and pointlike massive cores (see Sec. II) collapse on the same variable $(d)$, when estimated from the numerical solution of Eqs. (6), despite being closely related, do not necessarily coincide.

The functional dependence of $d_{\text {vor }}$ and $d_{\text {peak }}$ [extracted from the numerical solutions of system (6)] on $N_{b}$ is illustrated in Fig. 3 , together with the relation $d\left(N_{b}\right)$, obtained, in turn, by means of substitutions

$$
k=\frac{h}{m_{a}}, \quad \rho_{*}=\frac{N_{a} m_{a}}{\pi R^{2}}, \quad m=\frac{N_{b} m_{b}}{2}
$$

into Eq. (4). Relations (8) allow one to match the analytical model (4) based on pointlike vortices and pointlike massive cores with the actual parameters used to model the quantum fluids within the mean-field approach [see system (6)].

The agreement between the analytical prediction (yellow dotted line) and numerical results (blue solid and red dashed lines) is remarkably good, both qualitatively (same quasilinear functional dependence on $N_{b}$ ) and quantitatively (offset $<2 \%$ ). Moreover, we would like to mention that numerical results (namely, the slope and the vertical shift of the corresponding lines of Fig. 3) can be shown to further approach the analytical prediction upon increasing $N_{a}$ and/or diminishing $g_{b}$, two changes that result in narrower cores and, therefore, in a scenario where Eq. (4), based on our pointlike approximation, reliably describes the mixture vortex state (see Appendix for further details concerning the validity of the pointlike approximation).

To conclude this section, we present Fig. 4, where we illustrate the dependence of the equilibrium distance on the rotating frequency $\Omega$, in the case of $N_{b}=1000$. As is visible, there is a considerable agreement between the predictions ensuing from the pointlike model (3) [more specifically, from condition (4)] and the results extracted from the numerical solutions of eigensystem (6), in a rather large range of rotating frequencies. One can notice that the typical inverse propor-

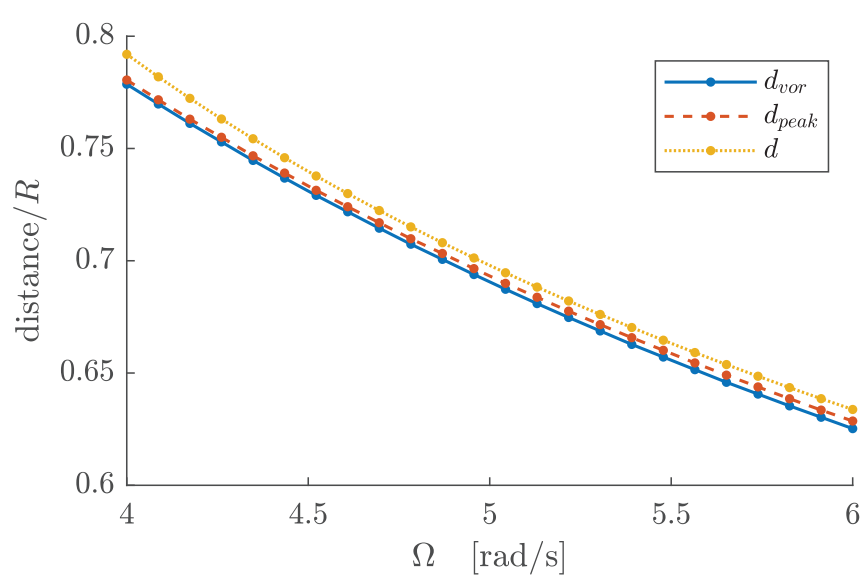

FIG. 4. Equilibrium distance as a function of the rotating frequency $\Omega$ : comparison between numerical ( $d_{\text {vor }}$ and $d_{\text {peak }}$ ) and analytical $(d)$ results. The following parameters have been used: $N_{a}=5 \times 10^{4}, N_{b}=1000, \Omega \in[4,6] \mathrm{rad} / \mathrm{s}, R=50 \mu \mathrm{m}, m_{a}=$ $3.82 \times 10^{-26} \mathrm{~kg}, m_{b}=6.48 \times 10^{-26} \mathrm{~kg}, g_{a}=52 \times\left(4 \pi \hbar^{2} a_{0}\right) / m_{a}$, $g_{b}=7.6 \times\left(4 \pi \hbar^{2} a_{0}\right) / m_{b}, g_{a b}=24.2 \times\left(2 \pi \hbar^{2} a_{0}\right) / m_{a b}, \ell_{z}=2 \mu \mathrm{m}$.

tionality of the equilibrium distance on the rotating frequency characterizing corotating massless vortices [17] is preserved in the dyamical regimes that we have been able to access by numerically solving eigensystem (6). In principle, as it is visible from the observation of formula (5); this inverse proportionality may be strongly altered by the presence of inertial terms which possible determine a diverging behavior when the two cores are so massive that $m \Omega^{2} \rightarrow k \rho_{*} \Omega$. Well before reaching this limit, where the inertial term $m \Omega^{2}$ would dominate over the standard term $k \rho_{*} \Omega$, the specific Bose-Bose mixture that we chose to employ cannot sustain a ground state where the mass $m$ may get too large as the associated number of species- $b$ atoms [see mapping (8)], together with the set of intra- and interspecies couplings would break the vortexbright soliton complexes and make other phase-separated configurations more favorable (see Appendix).

\section{B. Competition between centrifugal force and interspecies repulsion}

As already mentioned, $d_{\text {peak }}$, although closely related to $d_{\mathrm{vor}}$, is always slightly bigger than the latter. The motion of precession of the vortices around the center of the trap is responsible, in fact, for a centrifugal force on species- $b$ atoms which are, therefore, pushed outward. This tendency is only partially opposed by the repulsive interaction between the two quantum fluids and it is the competition between these two forces what determines the exact values of $d_{\mathrm{vor}}$ and

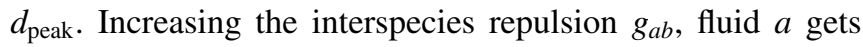
more impenetrable to species- $b$ atoms, which therefore prove to be more tightly bound within the valleys of $\left|\psi_{a}\right|^{2}$. As a result of this increased reaction to the centrifugal force, the difference $d_{\text {peak }}-d_{\text {vor }}$ is remarkably smaller, as illustrated in Fig. 5 (where $g_{a b}$ has been set 2.5 times bigger than the value used for Fig. 3).

\section{Stability beyond the immiscibility condition}

We remark that the results illustrated in this paper are obtained under the assumption that the two quantum fluids 


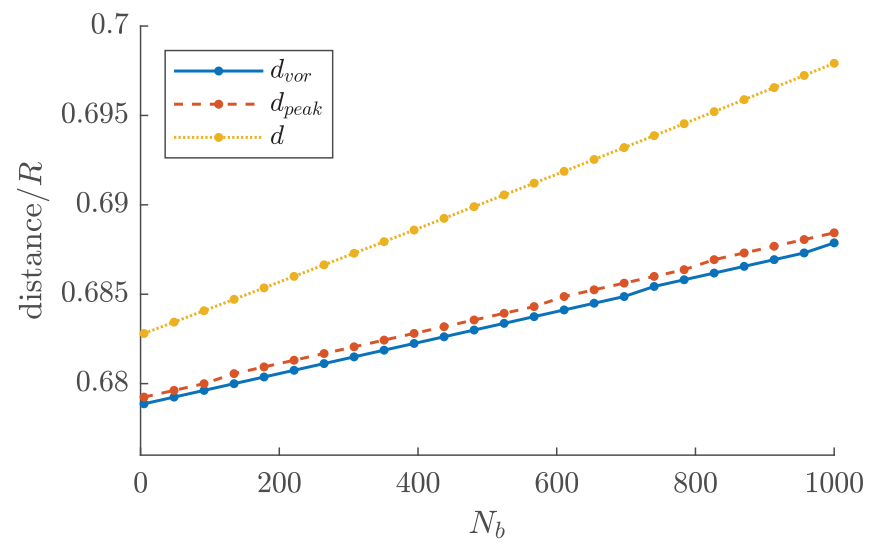

FIG. 5. Equilibrium distance: Comparison between numerical $\left(d_{\text {vor }}\right.$ and $\left.d_{\text {peak }}\right)$ and analytical $(d)$ results. For these simulations, parameter $g_{a b}$ is 2.5 times bigger than the one used for Fig. 3, all the others being unchanged.

are immiscible, meaning that their intra- and interspecies coupling parameters are such that $g_{a b} / \sqrt{g_{a} g_{b}}>1$. Actually, we should mention that vortex/bright-soliton complexes prove to be rather robust composite objects, as their stability extends also beyond the immiscible regime. We have verified this by numerically solving Eqs. (6), thus extending the results of Ref. [34], derived in the context of dark-bright soliton complexes, to the case of vortex/bright-soliton complexes. The obtained density distributions, $\left|\psi_{a}\right|^{2}$ and $\left|\psi_{b}\right|^{2}$, are qualitatively similar to the ones illustrated in Fig. 1 and obtained in the case of immiscible components. Of course, the difference between the miscible and the immiscible regime is that, in the latter case, bright solitons are more tightly confined within the vortex cores than in the former case. Moreover, due to the outward shift of the bright soliton from the vortex center, ensuing from the centrifugal force, the soliton itself, at a given time, feels an effective elliptical potential which, in turn, deforms its circular shape. Concerning our sample, which is immiscible, the deviation of the shape of bright solitons from a perfectly circular one can be neglected at first approximation, as the associated ellipticity, although being a decreasing function of $N_{b}$, is always $\approx 0.98$ in the whole considered range of $N_{b}$.

When the two quantum fluids are miscible, in fact, bright solitons manage to invade the majority component in a more significant way. As a consequence, they play the role of less rigid (i.e., softer) massive cores. If, on one hand, this circumstance extends the robustness of these composite objects to the case of miscible quantum fluids, on the other hand, in the miscible regime, our pointlike model (3) partially loses its validity, as the mass of the bright solitons is not concentrated in the vortices centers any more, but spreads and occupies more extended spatial regions.

\section{ANGULAR MOMENTUM OF VORTICES AND CORES}

This section is devoted to the analysis of the angular momentum of each component, an investigation that can offer a deeper insight into the physics of the system. In particular, we show that the two massive cores (made of species- $b$ atoms) orbit around the center of the trap, being dragged by the

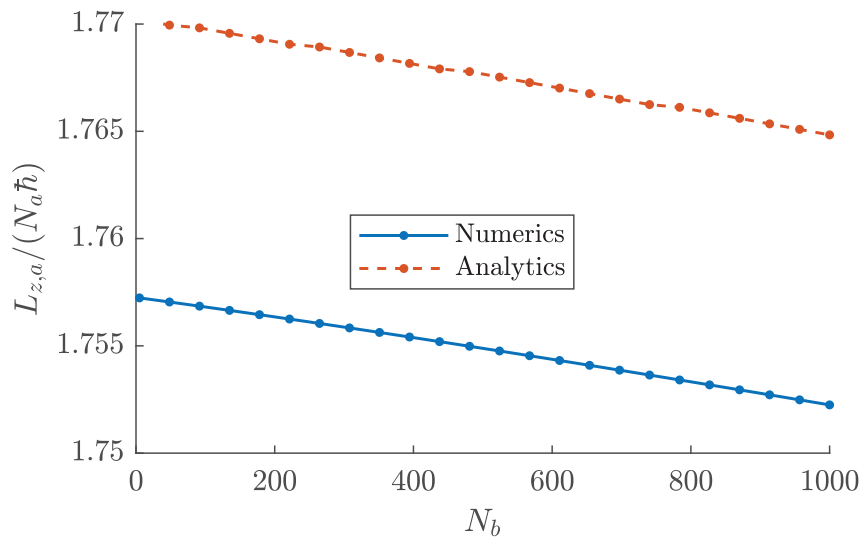

FIG. 6. Angular momentum (per particle, in units of $\hbar$ ) of condensate $a$ : Comparison between numerical [see Eq. (9)] and analytical [see Eq. (10)] results. The following parameters have been used: $N_{a}=5 \times 10^{4}, N_{b} \in[5,1000], \Omega=5 \mathrm{rad} / \mathrm{s}, R=50 \mu \mathrm{m}, m_{a}=$ $3.82 \times 10^{-26} \mathrm{~kg}, m_{b}=6.48 \times 10^{-26} \mathrm{~kg}, g_{a}=52 \times\left(4 \pi \hbar^{2} a_{0}\right) / m_{a}$, $g_{b}=7.6 \times\left(4 \pi \hbar^{2} a_{0}\right) / m_{b}, g_{a b}=24.2 \times\left(2 \pi \hbar^{2} a_{0}\right) / m_{a b}, \ell_{z}=2 \mu \mathrm{m}$.

motion of precession of the vortices. Nevertheless, they do not rotate, i.e., their orientation remains constant while they revolve.

\section{A. Angular momentum of condensate $a$}

The angular momentum (per particle, in units of $\hbar$ ) of condensate $a$ can be computed as

$$
\frac{\left\langle L_{z, a}\right\rangle}{N_{a} \hbar}=-i \int \psi_{a}^{*}\left(x \frac{\partial}{\partial y}-y \frac{\partial}{\partial x}\right) \psi_{a} d x d y .
$$

This quantity can be evaluated numerically from the solution of Eqs. (6).

On the other hand, it can also be estimated by means of a fully analytical approach. Along the same lines discussed in Ref. [56] (where the authors investigated the case of harmonic confinement), in fact, it is possible to derive the following expression:

$$
\frac{\tilde{L}_{z, a}}{N_{a} \hbar}=2 \int_{r_{\mathrm{vor}}}^{R} 2 \pi r \frac{1}{\pi R^{2}} d r=2\left[1-\left(\frac{r_{\mathrm{vor}}}{R}\right)^{2}\right],
$$

where $r_{\mathrm{vor}}:=d_{\mathrm{vor}} / 2$ constitutes the orbit radius.

As shown in Fig. 6, Eq. (10) well fits the numerical data obtained by means of Eq. (9), the mismatch being $<0.8 \%$. In this regard, it can be shown that the fitting accuracy further increases if one increases $N_{a}$ and/or decreases $g_{b}$, because, in this case, the pointlike approximation of vortices and cores gets increasingly valid (see Appendix).

\section{B. Angular momentum of condensate $b$}

The angular momentum (per particle, in units of $\hbar$ ) of component $b$ can be analogously computed as

$$
\frac{\left\langle L_{z, b}\right\rangle}{N_{b} \hbar}=-i \int \psi_{b}^{*}\left(x \frac{\partial}{\partial y}-y \frac{\partial}{\partial x}\right) \psi_{b} d x d y,
$$

a quantity that can be evaluated numerically on the basis of the solution of Eqs. (6). 
As already mentioned, the two species- $b$ cores orbit around the center of the trap but they do not rotate around their own centers of mass. To prove this statement, we proceed along three different lines.

\section{Mass current density in the rotating frame}

In the laboratory frame, it is possible to compute the mass current density $\vec{J}_{b}$ associated to $\psi_{b}$ [see Eq. (7)]. The corresponding vector field is illustrated in the middle right panel of Fig. 1. It is clear that the left (right) core is moving downward (upward), dragged by the anticlockwise motion of precession of the vortices. Due to the characteristic magnitude of $\left|\vec{J}_{b}\right|$, this plot does not allow one to understand whether the cores change their orientation or not along their orbit around the center of the trap. To circumvent this limitation, we have computed the species- $b$ mass current density in a (noninertial) frame rotating with the same angular velocity $\Omega$ distinguishing the motion of precession of the vortices. More specifically, in the rotating frame, $\vec{J}_{b, \text { rot }}$ reads

$$
\vec{J}_{b, \text { rot }}=m_{b}\left|\psi_{b}\right|^{2} \vec{v}_{b, \text { rot }},
$$

where $\vec{v}_{b, \text { rot }}=\vec{v}_{b}-\vec{V}$ with $\vec{v}_{b}=\frac{\vec{J}_{b}}{m_{b}\left|\psi_{b}\right|^{2}}$ and $\vec{V}=\vec{\Omega} \wedge \vec{r}$. $\vec{J}_{b}$ is numerically computed through Eq. (7). Notice also that, at $t=0,\left|\psi_{b}\right|^{2} \equiv\left|\psi_{b, \text { rot }}\right|^{2}$, thus justifying its use in Eq. (12). The result of this procedure is illustrated in the upper panel of Fig. 7 which shows that the two species- $b$ cores, when observed from the rotating frame, rotate around their respective centers of mass, with angular velocity $-\Omega$ (the minus sign being due to the clockwise direction).

On top of that, we have evidenced how these two cores rotate almost as if they were rigid bodies, meaning that the (absolute value of the) velocity field $\vec{v}_{b \text {,rot }}$ around each center of mass linearly increases with the distance $r_{C}$ from the respective center of mass (this is not in contrast with the irrotational property of quantum gases, as the new reference frame is not inertial). In view of the symmetry of the groundstate configuration depicted in Fig. 1, we refer to the left (right) center of mass when considering the velocity field in the half-plane $x<0(x>0)$. The lower panel of Fig. 7 shows the local angular velocity of species- $b$ cores when observed from the rotating frame. This quantity, defined as

$$
\tilde{\Omega}_{b, \text { rot }}(x, y)=-\frac{\left|\vec{v}_{b, \text { rot }}\right|}{r_{C}},
$$

[where $r_{C}$ is the distance of point $(x, y)$ from the left (right) center of mass when $x<0(x>0)]$ and takes the (almost) constant value $\approx-5 \mathrm{rad} / \mathrm{s}$ in the most part of the regions where $\left|\psi_{b}\right|^{2}$ is nonzero.

As an alternative indicator, to investigate the possible rotational properties of bright solitons, one could employ the vorticity distribution in the rotating frame $\vec{w}_{b \text {, rot }}=\vec{\nabla} \wedge \vec{v}_{b \text {, rot }}$. Apart from two Dirac-delta-like singularities exactly where phase singularities are (see lower right panel of Fig. 1), one would observe two quasiplateaus at $\left|\vec{w}_{b \text {, rot }}\right|=2 \tilde{\Omega}_{b \text {, rot }} \approx$ $-10 \mathrm{rad} / \mathrm{s}$. This circumstance is in great agreement with the fact that the vorticity distribution associated to a rigid body rotating with angular frequency $\Omega$ is uniform and equal to $2 \Omega$.

In conclusion, we have proved that, in the (noninertial) rotating frame, the two species- $b$ cores rotate around their
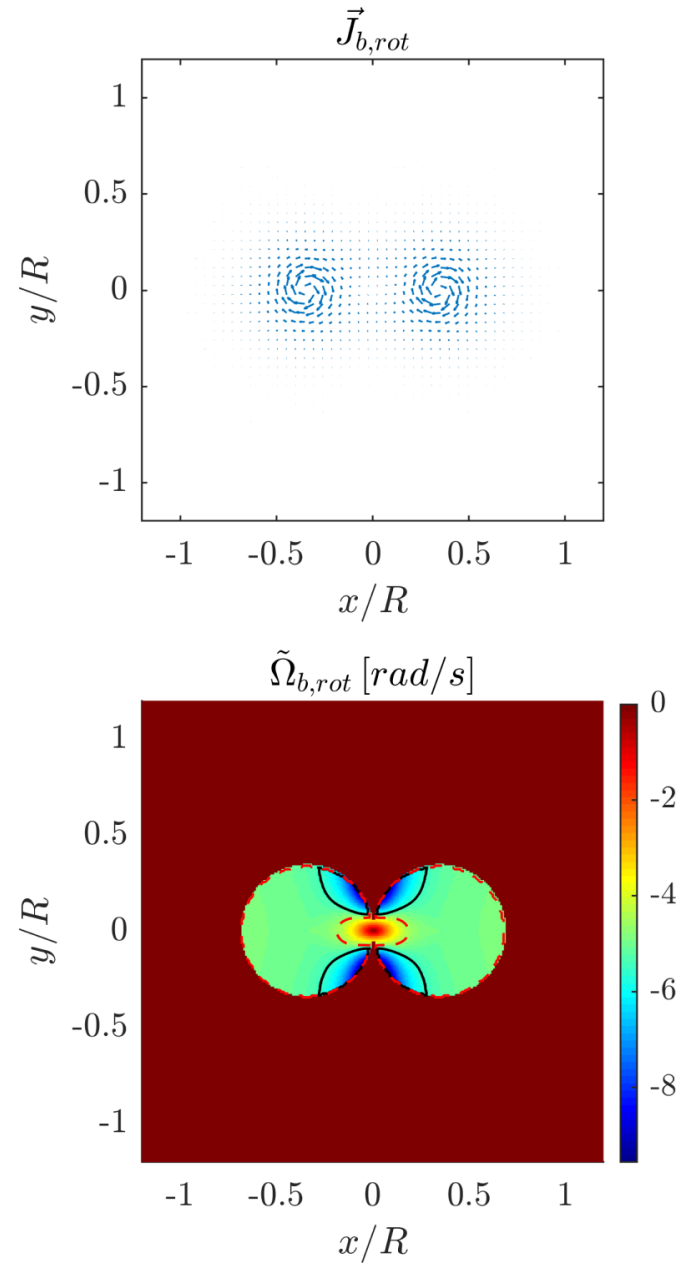

FIG. 7. Upper panel: Species- $b$ mass current density in the rotating frame [see Eq. (12) and the relevant explanation]: One can appreciate that the two cores rotate clockwise. Lower panel: Species$b$ local angular velocity, as defined by Eq. (13); The solid black and red dashed lines correspond to $\widetilde{\Omega}_{b, \text { rot }}=-5 \pm 0.5 \mathrm{rad} / \mathrm{s}$ and have been drawn to illustrate that the two species- $b$ cores indeed rotate as two (almost) rigid bodies (see discussion in the main text). The following parameters have been used: $N_{a}=5 \times 10^{4}, N_{b}=$ $10^{3}, \Omega=5 \mathrm{rad} / \mathrm{s}, R=50 \mu \mathrm{m}, m_{a}=3.82 \times 10^{-26} \mathrm{~kg}, m_{b}=6.48 \times$ $10^{-26} \mathrm{~kg}, g_{a}=52 \times\left(4 \pi \hbar^{2} a_{0}\right) / m_{a}, g_{b}=7.6 \times\left(4 \pi \hbar^{2} a_{0}\right) / m_{b}, g_{a b}=$ $24.2 \times\left(2 \pi \hbar^{2} a_{0}\right) / m_{a b}, \ell_{z}=2 \mu \mathrm{m}$.

respective centers of mass with (almost uniform) angular velocity $-\Omega$. This allows us to conclude that they keep their orientation fixed when observed from the laboratory frame.

\section{Analytical estimate of the angular momentum}

To corroborate what elucidated in the previous paragraph, we show that the functional dependence of quantity (11) on model parameter $N_{b}$ can be well fitted by the semianalytical model

$$
\frac{\tilde{L}_{z, b}}{N_{b} \hbar} \approx \frac{\tilde{\mathcal{L}}_{O, b}}{N_{b} \hbar}+\frac{\tilde{\mathcal{S}}_{C_{L}, b}}{N_{b} \hbar}+\frac{\tilde{\mathcal{S}}_{C_{R}, b}}{N_{b} \hbar},
$$

where

$$
\frac{\tilde{\mathcal{L}}_{O, b}}{N_{b} \hbar}=\frac{\Omega}{\hbar} \int m_{b}\left|\psi_{b}\right|^{2} r^{2} d x d y
$$




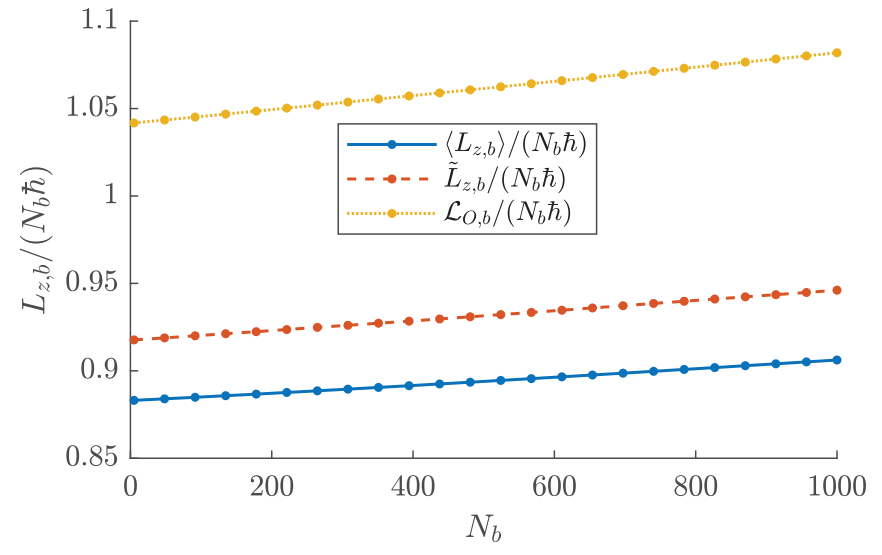

FIG. 8. Angular momentum (per particle, in units of $\hbar$ ) of component $b$ : Comparison between numerical [solid blue line, associated to Eq. (11)] and semianalytical [red dashed line, corresponding to Eq. (14)] results [the yellow dotted line, associated to Eq. (15) represents the angular momentum of the system if its motion was a pure revolution around the center of the trap $O]$. The following parameters have been used: $N_{a}=5 \times 10^{4}, N_{b} \in[5,1000], \Omega=5 \mathrm{rad} / \mathrm{s}, R=50 \mu \mathrm{m}, m_{a}=$ $3.82 \times 10^{-26} \mathrm{~kg}, m_{b}=6.48 \times 10^{-26} \mathrm{~kg}, g_{a}=52 \times\left(4 \pi \hbar^{2} a_{0}\right) / m_{a}$, $g_{b}=7.6 \times\left(4 \pi \hbar^{2} a_{0}\right) / m_{b}, g_{a b}=24.2 \times\left(2 \pi \hbar^{2} a_{0}\right) / m_{a b}, \ell_{z}=2 \mu \mathrm{m}$.

and where terms

$$
\begin{aligned}
\frac{\tilde{\mathcal{S}}_{C_{L}, b}}{N_{b} \hbar} & =-\frac{\Omega}{\hbar} \int m_{b}\left|\psi_{b}\right|^{2} \Theta(-x) r_{C_{L}}^{2} d x d y, \\
\frac{\tilde{\mathcal{S}}_{C_{R}, b}}{N_{b} \hbar} & =-\frac{\Omega}{\hbar} \int m_{b}\left|\psi_{b}\right|^{2} \Theta(x) r_{C_{R}}^{2} d x d y
\end{aligned}
$$

are introduced to take into account that, in the laboratory frame, the two species- $b$ cores revolve but keep their orientation fixed [Heaviside functions $\Theta(-x)$ and $\Theta(x)$ allow one to select the left and the right cores, respectively]. The integrals in expressions (15)-(17) represent three moments of inertia corresponding, respectively, to the anticlockwise revolution of the whole system around the center of the trap $O$, and to the effective clockwise rotation of the left (right) core around its own center of mass $C_{L}\left(C_{R}\right)$ [in this regard, $r^{2}:=$ $x^{2}+y^{2}, r_{C_{\alpha}}^{2}:=\left(x-x_{C_{\alpha}}\right)^{2}+\left(y-y_{C_{\alpha}}\right)^{2}$, with $\left.\alpha=L, R\right]$. The latter effective motions indeed compensate for the fact that a pure motion of revolution [captured by Eq. (15)] would determine a change in the orientation of the cores along the circular orbit. Figure 8 shows a very good agreement between Eq. (11) and formula (14), the error being always $<4 \%$.

\section{Observation of the associated phase field}

As illustrated in the lower right panel of Fig. 1, the phase field associated to $\psi_{b}, \theta_{b}$, features two singularities far from the centers of bright solitons. If one considers a closed path $\gamma$ encircling one of the bright solitons, since no singularities of $\theta_{b}$ are surrounded by it, the circulation $\mathcal{C}_{\gamma}\left[\vec{v}_{b}\right]$ of the velocity field $\vec{v}_{b}$ along $\gamma$ must be zero (see Sec. III). This implies that both bright solitons do not rotate around their own axes (in the laboratory inertial frame). On the other hand, these singularities in $\theta_{b}$ indeed play an important role because the corresponding velocity field $\vec{v}_{b}=\frac{\hbar}{m_{b}} \nabla \theta_{b}$ is what determines the collective precession motion of species- $b$ bosons.

In summary, we notice that the interspecies repulsive coupling $g_{a b}$ is the interaction underlining the dragging of species- $b$ cores by species- $a$ vortices, which therefore exhibit the same motion of precession. In spite of this precession, species- $b$ cores keep their orientation constant (in the inertial frame of the laboratory). Due to the irrotational properties of quantum fluids, in fact, a solitonlike distribution cannot be put in rotation around its own axis (as if it was a rigid body) without creating a phase singularity at its center. On the other hand, the creation of such a phase singularity would turn the soliton-like original distribution into a vortical object. To conclude this section, we would like to mention the possible existence of the Andreev-Bashkin effect [57-60], according to which, the mass current density $\vec{J}_{i}$ (with $i=a, b$ ) in one species will depend, in general, also on the velocity $\vec{v}_{j}$ (with $j=b, a)$ of the other species. In other words, the condensate density $\rho_{i j}$ is a nondiagonal matrix, a circumstance which implies the relations

$$
\vec{J}_{a}=\rho_{a a} \vec{v}_{a}+\rho_{a b} \vec{v}_{b}, \quad \vec{J}_{b}=\rho_{b a} \vec{v}_{a}+\rho_{b b} \vec{v}_{b}
$$

At the microscopic scale, this drag between mass current densities comes from the formation of quasiparticles with nonzero content of mass for either of the two components [60]. As a consequence, the transport properties of the two quantum fluids turn out to be coupled: The flow of one species influences the mass transport in the other species [57]. This effect is known to be rather elusive [60] and, for our sample condition, we expect it to be negligible, as the off-diagonal matrix elements $\rho_{a b}$ and $\rho_{b a}$, which depend on the the overlap between $\psi_{a}$ and $\psi_{b}$, should be small, given that the two discussed fluids are immiscible. In view of its complexity, the possible presence of this effect in the discussed system of orbiting vortex/bright-soliton complexes will be analyzed in a future work.

\section{VORTEX HEALING LENGTHS AND SIZE OF THE MASSIVE CORES}

The presence of species- $b$ massive cores within species$a$ vortices affects the healing length of the latter. The intraspecies repulsive interaction, in fact, tends to enlarge the cores which, in turn, tend to swell (from the inside) the profile of the vortices because of the interspecies repulsive coupling. Flipping the perspective, the expansion of the cores is dammed by the species- $a$ fluid, which plays the role of an effective confining potential for species- $b$ atoms.

In the attempt to estimate the equilibrium healing length $\xi_{a}$ of species- $a$ vortices and the equilibrium characteristic size of species- $b$ cores, $\sigma_{b}$, we present the following heuristic equations:

$$
\begin{gathered}
\frac{\hbar^{2}}{2 m_{a}} \frac{1}{\xi_{a}^{2}}=+g_{a} n_{a}-g_{a b} n_{a} n_{b} \pi\left(\sigma_{b}^{2}-\xi_{a}^{2}\right) \ell_{z}, \\
\frac{\hbar^{2}}{2 m_{b}} \frac{1}{\sigma_{b}^{2}}=-g_{b} n_{b}+g_{a b} n_{a} n_{b} \pi\left(\sigma_{b}^{2}-\xi_{a}^{2}\right) \ell_{z},
\end{gathered}
$$


where

$$
n_{a}=\frac{N_{a}}{\pi r_{\mathrm{box}}^{2} \ell_{z}}, \quad n_{b}=\frac{N_{b} / 2}{\pi \sigma_{b}^{2} \ell_{z}} .
$$

Notice that the the first equation of system (18) reduces, in the case of no interspecies interaction, to

$$
\xi_{a, 0}=\sqrt{\frac{\hbar^{2}}{2 m_{a} g_{a} n_{a}}},
$$

the well-known formula derived in the context of singlespecies vortices [61]. Similarly, the second equation, if $g_{a b}=$ 0 , gets structurally similar to formula

$$
\sigma_{b, 0}=\sqrt{\frac{\hbar^{2}}{2 m_{b}\left|g_{b}\right| n_{b, 0}}},
$$

giving the characteristic size of a soliton in the case of attractive interactions [61] (of course, in this context, $n_{b, 0}$ represents the central density). The extra term $g_{a b} n_{a} n_{b} \pi\left(\sigma_{b}^{2}-\xi_{a}^{2}\right) \ell_{z}$ is introduced to take into account the interspecies repulsion, an interaction that manifests only in those regions where $\psi_{a}$ and $\psi_{b}$ overlap, i.e., only in the two annuli centered in the vortices' centers and whose outer and inner radii are $\sigma_{b}$ and $\xi_{a}$, respectively.

To compare the predictions provided by equations (18) with the values extracted from the numerical solutions of system (6), one has to give the operational definition of vortex healing length and core characteristic size. From the numerical side, with reference to Fig. 2, we agree to measure the half width at half maximum of the valley of $\left|\psi_{a}\right|^{2}, \lambda_{a}$, and the half width at half maximum of the peak of $\left|\psi_{b}\right|^{2}$, $\lambda_{b}$. From the analytical side, the estimates of quantities $\lambda_{a}$ and $\lambda_{b}$ are given by the solutions of system (18), $\xi_{a}$ and $\sigma_{b}$, multiplied by two suitable constant conversion factors, 1.30 and 1.15 , respectively, which are determined from their numerical counterpart in the case $N_{b}=1$, that means in a scenario where species- $b$ cores have a negligible impact on species- $a$ vortices.

As illustrated in Fig. 9, Eqs. (18) well capture the functional dependence of $\lambda_{a}$ and of $\lambda_{b}$ on $N_{b}$.

\section{CONCLUDING REMARKS}

In this paper, we have investigated a notable class of configurations exhibited by a bosonic immiscible binary mixture loaded in a boxlike circular trap, namely, minimumenergy states where species- $b$ atoms are trapped within the vortex cores of species- $a$ fluid. Both within a fully analytical framework and by means of a systematic analysis of the numerical solutions of the associated two coupled GPEs, we have shown that the presence of massive cores alters the equilibrium distance distinguishing the motion of precession of the vortex pair. Interestingly, for the considered choices of model parameters (repulsive intra- and interspecies interactions such that, in the homogeneous case, the miscibility condition $g_{a b}<\sqrt{g_{a} g_{b}}$ is not met) the dynamical mean-field picture of the mixture has been shown to reduce to much simpler effective equations exhibiting an evident Lorentzlike magnetic form, where massive vortices play the role of massive charges confined on a plane and subject to a magnetic
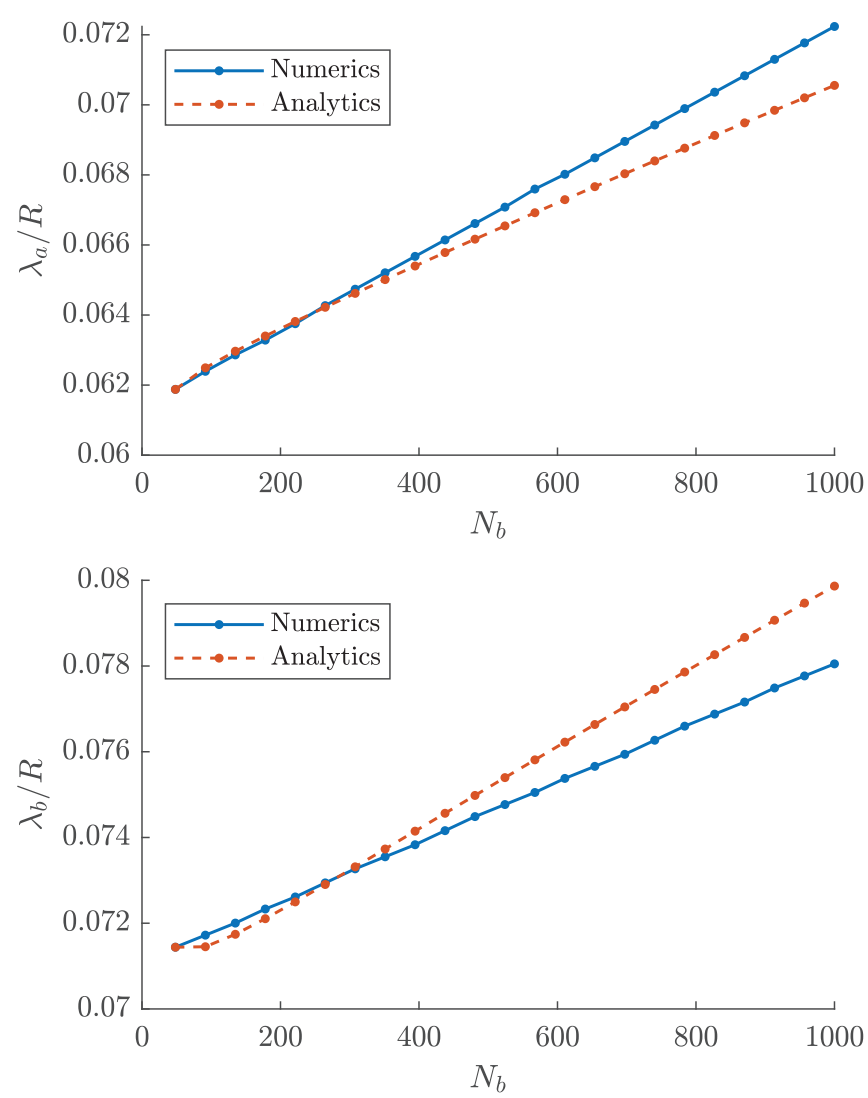

FIG. 9. Upper (lower) panel: Comparison between the numerically determined and analytically estimated half width half maximum, $\lambda_{a}$, of species- $a$ vortices (of species- $b$ cores, $\lambda_{b}$ ). The following parameters have been used: $N_{a}=5 \times 10^{4}, N_{b} \in[1,1000], \Omega=$ $5 \mathrm{rad} / \mathrm{s}, R=50 \mu \mathrm{m}, m_{a}=3.82 \times 10^{-26} \mathrm{~kg}, m_{b}=6.48 \times 10^{-26} \mathrm{~kg}$, $g_{a}=52 \times\left(4 \pi \hbar^{2} a_{0}\right) / m_{a}, \quad g_{b}=7.6 \times\left(4 \pi \hbar^{2} a_{0}\right) / m_{b}, \quad g_{a b}=24.2 \times$ $\left(2 \pi \hbar^{2} a_{0}\right) / m_{a b}, \ell_{z}=2 \mu \mathrm{m}$.

field. Species- $b$ cores, in turn, are dragged by fluid $a$ and thus follow their same motion of precession around the trap center; nevertheless, while orbiting, they keep their orientation constant, meaning that there is no tangential entrainment between the two fluids. We have also derived, in the context of the Thomas-Fermi approximation, a simple formula to estimate the angular momentum of condensate $a$ and we have shown, by means of a suitable change of reference frame, that species$b$ cores effectively behave as two rigid bodies. Eventually, we have introduced a system of heuristic but effective equations to estimate the characteristic size of vortices and cores hosted therein.

\section{ACKNOWLEDGMENTS}

The authors wish to thank M. Pi, A. Gallemí and Y. Bidasyuk for their valuable help in setting up the numerical simulations. V.P. is indebted to F. Minardi for useful discussions about trapping techniques. M.G. and R.M. acknowledge financial support from the Spanish MINECO and Fondo Europeo de Desarrollo Regional (FEDER, EU) under Grant No. FIS2017-87801-P, and from Generalitat de Catalunya Grant No. 2017SGR533. 

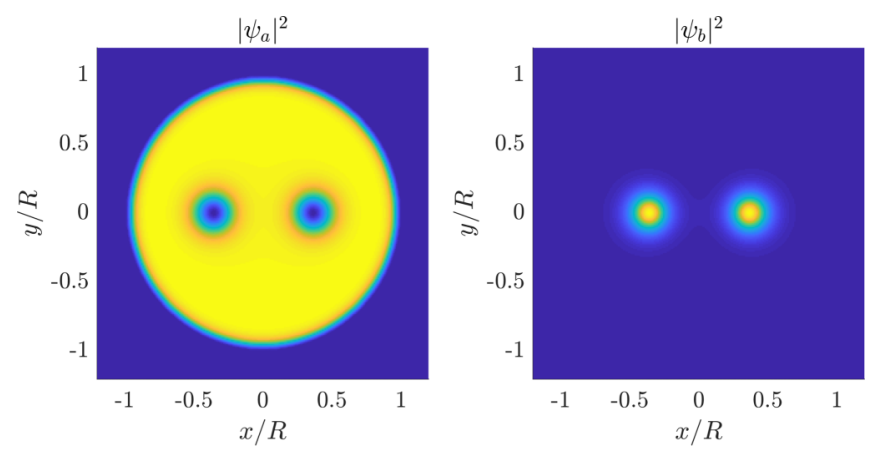

FIG. 10. Minimum-energy solution of eigensystem (6) for the same model parameters used in Fig. 1, except that the number of species- $b$ atoms is now $N_{b}=5000$. Vortex cores and bright solitons are considerably enlarged with respect to those ones shown in Fig. 1 and obtained for $N_{b}=1000$.

\section{APPENDIX}

In this Appendix, we comment on the validity of the presented pointlike model (3). The latter is based on the assumption that the equilibrium healing length of species- $a$ vortices [variable $\xi_{a}$ in formulas (18)] is much smaller than the trap radius $R$. On the other hand, increasing the number of species- $b$ atoms, the two bright solitons get increasingly massive and increasingly wide (due to the fact that the intraspecies coupling $g_{b}$ is repulsive). Therefore, the presence of massive cores deforms the original shape of vortices (see Fig. 2), thus increasing $\xi_{a}$ (see Fig. 9).

Figure 10 shows the (square modulus of the) solutions of eigensystem (6) in the case of $N_{b}=5000$, a value which is five

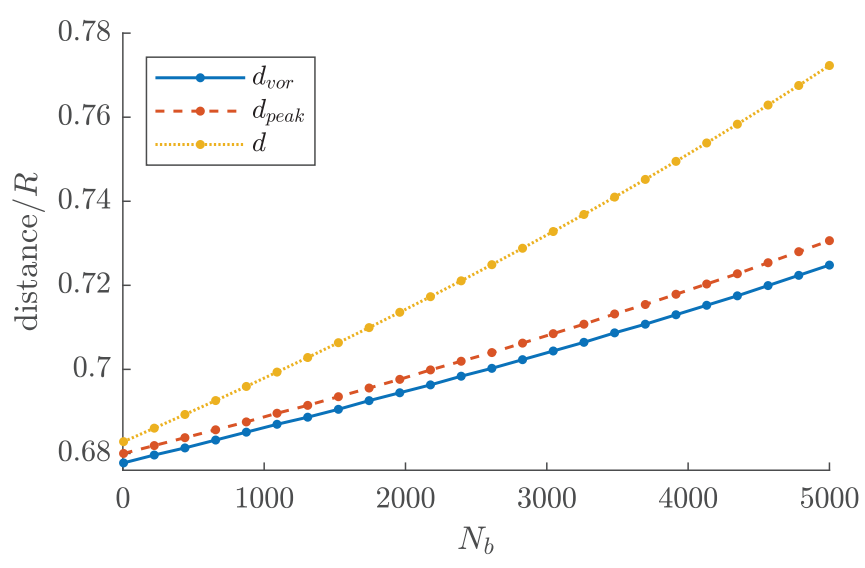

FIG. 11. Equilibrium distance: comparison between numerical ( $d_{\mathrm{vor}}$ and $d_{\text {peak }}$ ) and analytical $(d)$ results. The same model parameters used in Fig. 3 have been employed, except that the number of species- $b$ atoms is swept in the range $N_{b} \in[5,5000]$.

times bigger than the one used for Fig. 1. By comparing the latter figures, one can appreciate the fact that neglecting the finite size of vortex cores may be not fully justified when $N_{b}$ gets reasonably large. In this circumstance, in fact, the predictions ensuing from the pointlike model (3) [more specifically, from the solution of equation (4)] may deviate from the results extracted from the numerical solutions of eigensystem (6) (see Fig. 11). If one further increases $N_{b}$, the deforming effect of bright solitons on the vortex profile will be so relevant that symbiotic vortex-bright soliton complexes will cease to exist and different phase-separated configurations will take over.
[1] L. Onsager, Il Nuovo Cimento (1943-1954) 6, 279 (1949).

[2] L. M. Pismen and L. M. Pismen, Vortices in Nonlinear Fields: From Liquid Crystals to Superfluids, from Non-equilibrium Patterns to Cosmic Strings (Oxford University Press, Oxford, UK, 1999), Vol. 100.

[3] R. P. Feynman, in Progress in Low Temperature Physics, Vol. 1 (Elsevier, North-Holland, Amsterdam, 1955), pp. 17-53.

[4] R. J. Donnelly, Quantized Vortices in Helium II (Cambridge University Press, Cambridge, 1991), Vol. 2.

[5] M. Brambilla, L. A. Lugiato, V. Penna, F. Prati, C. Tamm, and C. O. Weiss, Phys. Rev. A 43, 5114 (1991).

[6] I. Carusotto and C. Ciuti, Rev. Mod. Phys. 85, 299 (2013).

[7] G. Blatter, M. V. Feigel'man, V. B. Geshkenbein, A. I. Larkin, and V. M. Vinokur, Rev. Mod. Phys. 66, 1125 (1994).

[8] R. P. Huebener, Magnetic Flux Structures in Superconductors: Extended Reprint of a Classic Text (Springer Science \& Business Media, Berlin, 2013), Vol. 6.

[9] Vortex dynamics and quantum effects in Josephson junction arrays are reviewed in K.-H. Wagenblast, R. Fazio, A. van Otterlo, G. Schön, D. Zappalá, and G. T. Zimanyi, Physica B 222, 336 (1996), Proceedings of the ICTP Workshop on Josephson Junction Arrays, edited by H. A. Cerdeira and S. R. Shenoy.

[10] R. Fazio and H. Van Der Zant, Phys. Rep. 355, 235 (2001); V. M. Kaurov and A. B. Kuklov, Phys. Rev. A 73, 013627
(2006); J. A. Gil Granados, A. Muñoz Mateo, M. Guilleumas, and X. Viñas, New J. Phys. 21, 043036 (2019).

[11] C. F. Barenghi, R. J. Donnelly, and W. Vinen, Quantized Vortex Dynamics and Superfluid Turbulence (Springer Science \& Business Media, Berlin, 2001), Vol. 571.

[12] J. M. Kosterlitz and D. J. Thouless, J. Phys. C 6, 1181 (1973).

[13] R. Y. Chiao, A. Hansen, and A. A. Moulthrop, Phys. Rev. Lett. 54, 1339 (1985).

[14] A. L. Fetter, Phys. Rev. 162, 143 (1967).

[15] M. Rasetti and T. Regge, Physica A 80, 217 (1975).

[16] V. Penna, M. Rasetti, and M. Spera, Contemp. Math. 219, 173 (1998).

[17] A. L. Fetter, Rev. Mod. Phys. 81, 647 (2009).

[18] R. Dum, J. I. Cirac, M. Lewenstein, and P. Zoller, Phys. Rev. Lett. 80, 2972 (1998).

[19] D. Butts and D. Rokhsar, Nature 397, 327 (1999).

[20] F. Dalfovo and S. Stringari, Phys. Rev. A 53, 2477 (1996).

[21] L. Pismen and J. Rubinstein, Physica D 47, 353 (1991).

[22] M. Cozzini and S. Stringari, Phys. Rev. A 67, 041602(R) (2003).

[23] Y. Castin and R. Dum, Eur. Phys. J. D 7, 399 (1999).

[24] M. R. Matthews, B. P. Anderson, P. C. Haljan, D. S. Hall, C. E. Wieman, and E. A. Cornell, Phys. Rev. Lett. 83, 2498 (1999). 
[25] A. Recati, F. Zambelli, and S. Stringari, Phys. Rev. Lett. 86, 377 (2001).

[26] S. Sinha and Y. Castin, Phys. Rev. Lett. 87, 190402 (2001).

[27] K. W. Madison, F. Chevy, V. Bretin, and J. Dalibard, Phys. Rev. Lett. 86, 4443 (2001).

[28] R. Onofrio, C. Raman, J. M. Vogels, J. R. Abo-Shaeer, A. P. Chikkatur, and W. Ketterle, Phys. Rev. Lett. 85, 2228 (2000).

[29] D. R. Scherer, C. N. Weiler, T. W. Neely, and B. P. Anderson, Phys. Rev. Lett. 98, 110402 (2007).

[30] P. G. Drazin and R. S. Johnson, Solitons: An Introduction, Vol. 2 (Cambridge University Press, Cambridge, UK, 1989).

[31] S. Burger, L. D. Carr, P. Öhberg, K. Sengstock, and A. Sanpera, Phys. Rev. A 65, 043611 (2002).

[32] D. Frantzeskakis, J. Phys. A: Math. Theor. 43, 213001 (2010).

[33] C. Becker, S. Stellmer, P. Soltan-Panahi, S. Dörscher, M. Baumert, E.-M. Richter, J. Kronjäger, K. Bongs, and K. Sengstock, Nat. Phys. 4, 496 (2008).

[34] C. Hamner, J. J. Chang, P. Engels, and M. A. Hoefer, Phys. Rev. Lett. 106, 065302 (2011).

[35] S. Middelkamp, J. Chang, C. Hamner, R. Carretero-González, P. Kevrekidis, V. Achilleos, D. Frantzeskakis, P. Schmelcher, and P. Engels, Phys. Lett. A 375, 642 (2011).

[36] T. Busch and J. R. Anglin, Phys. Rev. Lett. 87, 010401 (2001).

[37] V. M. Pérez-García and J. B. Beitia, Phys. Rev. A 72, 033620 (2005).

[38] B. P. Anderson, P. C. Haljan, C. E. Wieman, and E. A. Cornell, Phys. Rev. Lett. 85, 2857 (2000).

[39] K. J. H. Law, P. G. Kevrekidis, and L. S. Tuckerman, Phys. Rev. Lett. 105, 160405 (2010).

[40] K. Mukherjee, K. Mukherjee, S. Mistakidis, P. G. Kevrekidis, and P. Schmelcher, J. Phys. B: Atom. Mol. Opt. Phys. (2020), doi: 10.1088/1361-6455/ab678d.

[41] A. Gallemí, L. P. Pitaevskii, S. Stringari, and A. Recati, Phys. Rev. A 97, 063615 (2018).

[42] E. G. Charalampidis, P. G. Kevrekidis, D. J. Frantzeskakis, and B. A. Malomed, Phys. Rev. E 94, 022207 (2016).
[43] P. Kuopanportti, S. Bandyopadhyay, A. Roy, and D. Angom, Phys. Rev. A 100, 033615 (2019).

[44] M. Pola, J. Stockhofe, P. Schmelcher, and P. G. Kevrekidis, Phys. Rev. A 86, 053601 (2012).

[45] S. Bandyopadhyay, A. Roy, and D. Angom, Phys. Rev. A 96, 043603 (2017).

[46] P. G. Saffman, Vortex Dynamics (Cambridge University Press, Cambridge, UK, 1992).

[47] K. Henderson, C. Ryu, C. MacCormick, and M. Boshier, New J. Phys. 11, 043030 (2009).

[48] C. Muldoon, L. Brandt, J. Dong, D. Stuart, E. Brainis, M. Himsworth, and A. Kuhn, New J. Phys. 14, 073051 (2012).

[49] G. Gauthier, I. Lenton, N. M. Parry, M. Baker, M. J. Davis, H. Rubinsztein-Dunlop, and T. W. Neely, Optica 3, 1136 (2016).

[50] V. Penna, in Quantized Vortex Dynamics and Superfluid Turbulence, edited by C. F. Barenghi, R. J. Donnelly, and W. F. Vinen (Springer-Verlag, Berlin 2001), pp 445-452.

[51] V. Penna, Phys. Rev. B 59, 7127 (1999).

[52] T. A. Schulze, T. Hartmann, K. K. Voges, M. W. Gempel, E. Tiemann, A. Zenesini, and S. Ospelkaus, Phys. Rev. A 97, 023623 (2018).

[53] A. Richaud, A. Zenesini, and V. Penna, Sci. Rep. 9, 6908 (2019).

[54] F. Coppens, F. Ancilotto, M. Barranco, N. Halberstadt, and M. Pi, Phys. Chem. Chem. Phys. 19, 24805 (2017).

[55] Computational resources provided by HPC@POLITO (http://www.hpc.polito.it).

[56] M. Guilleumas and R. Graham, Phys. Rev. A 64, 033607 (2001).

[57] A. Andreev and E. Bashkin, Sov. Phys. JETP 42, 164 (1976).

[58] E. Babaev, Phys. Rev. D 70, 043001 (2004).

[59] S. Shevchenko and D. Fil, J. Exp. Theor. Fisica 105, 135 (2007).

[60] J. Nespolo, G. E. Astrakharchik, and A. Recati, New J. Phys. 19, 125005 (2017).

[61] L. Pitaevskii and S. Stringari, Bose-Einstein Condensation and Superfluidity, Vol. 164 (Oxford University Press, Oxford, 2016). 